\title{
Cervical re-injection to improve sentinel lymph node detection in endometrial cancer
}

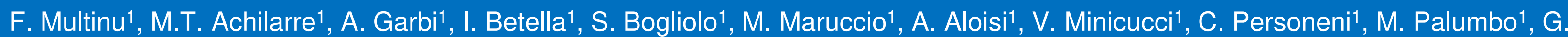
Aletti ${ }^{1}, 2$, N. Colombo ${ }^{1,3}$, A. Maggioni ${ }^{1}$, V. Zanagnolo ${ }^{1}$.

${ }^{1}$ IEO, European Institute of Oncology IRCCS, Department of Gynecologic Oncology, Milan, Italy.

2 Università degli Studi di Milano, Dipartimento di Oncologia ed Emato-Oncologia, Milan, Italy.

${ }_{3}^{3}$ University of Milan-Bicocca, Dipartimento di Scienze Chirurgiche - Facoltà di Medicina e Chirurgia, Milan, Italy

\section{Background}

Current guidelines for the treatment of EC recommend a comprehensive surgical staging including both pelvic and para-aortic lymphadenectomy (LND) [2], however many controversies on the role of surgical staging still exist among gynecologic oncologists [3].

- With the intent to find a balance between risk and harms of systematic pelvic and para-aortic LND vs. no nodal staging, sentinel lymph node (SLN) mapping (which has been demonstrated to be accurate in identifying the patients with lymphatic dissemination [4]) is gaining acceptance between gynecologists and was recently included within the National Comprehensive Cancer Network guidelines as a valuable option in the management of EC [5].

\section{Objectives}

- To evaluate the impact of cervical re-injection on the detection rate of fluorescence-guided sentinel lymph node (SLN) mapping in endometrial cancer (EC) patients undergoing robotic-assisted surgical staging

\section{Results}

- Of the 107 patients undergoing robotic-assisted surgical staging for EC during the study period, 7 cases with no detection or unilateral detection who did not underwent reinjection were excluded.

- Among the remaining 100 patients, after a single injection the overall detection rate was $98 \%(95 \% \mathrm{Cl}, 92.2-99.6 \%)$ with a $69 \%(95 \% \mathrm{Cl}, 58.8-77.7 \%)$ of bilateral detection rate.

- After re-injection, overall and bilateral detection rate were $100 \%(95 \% \mathrm{Cl}, 95.3-100 \%)$ and $91 \%(95 \% \mathrm{Cl}, 8.32-95.5 \%)$, respectively.

Clinicopathologic characteristics

\section{Methods}

- From April, 12017 to December, 312018 patients undergoing robotic-assisted surgery for apparently earlystage EC at our Institution were prospectively treated with SLN mapping using indocyanine green (ICG) accordingly to the Memorial Sloan Kettering Cancer Center (MSKCC) surgical algorithm.

- As per MSKCC algorithm, four $\mathrm{mL}(0,50 \mathrm{mg} / \mathrm{mL})$ of ICG were injected into the cervical submucosa and stroma, at the 3 and 9 o'clock positions ( $1 \mathrm{~mL}$ each).

- In case of either no detection or unilateral detection, cervical re-injection was performed followings the same steps as previously described.

- Overall (successful mapping of at least one hemipelvis) and bilateral detection were evaluated pre- and post-reinjection.
ENROLLED PATIENTS $(n=100)$
ROBOTIC SURGERY EARLY STAGE EC $(n=107)$

CTION \& NO RE-INJECTION $(n=7)$

\begin{tabular}{|l|c|}
\hline Age (years) (IC 95\%) & $60,5(58,1-62,9)$ \\
\hline BMI (kg/m²) (IC 95\%) & $26,9(25,6-28,2)$ \\
\hline Histology (final pathology) & \\
Endometrioid & 90 \\
Serous & 1 \\
Mucinous & 5 \\
Clear cell & 3 \\
Undifferentiated & 1 \\
\hline Grade (final pathology) & \\
$\mathbf{1}$ & 41 \\
$\mathbf{2}$ & 35 \\
$\mathbf{3}$ & 24 \\
\hline FIGO Stage (final pathology) & \\
Ia & 73 \\
Ib & 12 \\
II & 4 \\
IIIa & 2 \\
\hline
\end{tabular}

SLN detection rate

\begin{tabular}{|c|c|c|}
\hline \multicolumn{3}{|c|}{ SLN detection rate } \\
\hline & $\begin{array}{c}\text { 1st ICG injection } \\
(\%, \mathbf{C I} \text { 95\%) }\end{array}$ & $\begin{array}{c}\text { ICG reinjection } \\
(\mathbf{\%}, \mathbf{C I} \mathbf{9 5} \%)\end{array}$ \\
\hline Unilateral & $98 \%(92.2-99.6)$ & $100 \%(95.3-100)$ \\
\hline Bilateral & $69 \%(58.8-77.7)$ & $\mathbf{9 1 \%}(\mathbf{8 3 . 2 - 9 5 . 5 )}$ \\
\hline
\end{tabular}

\section{Conclusions}

In the case of no detection or unilateral sentinel lymph node detection, cervical re-injection of ICG can increase overall and bilateral detection rate, thus decreasing the number of patients requiring a complete bilateral or side-specific lymphadenectomy

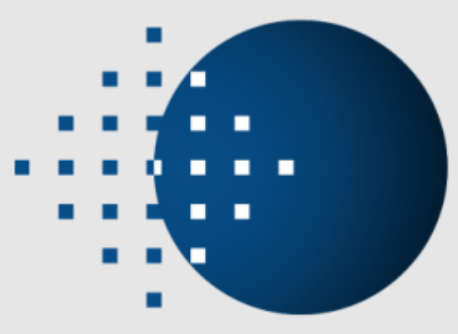

